\title{
Hemoglobinas anormais e dificuldade diagnóstica
}

\author{
Guilherme G. Leoneli ${ }^{1}$ \\ Rodrigo E. Imperial ${ }^{2}$ \\ Daniela P. Marchi-Salvador ${ }^{3}$ \\ Paulo C. Naoum ${ }^{4}$ \\ Cláudia R. Bonini-Domingos ${ }^{5}$
}

\begin{abstract}
As hemoglobinas humanas, com padrão de herança definido gen eticamente, a presentam variações polimórficascaracterísticas dentro de nossa população, na dependência dos grupos raciais que formam cada região. Aparecem sob a forma de variantes de hemoglobinas ou talassemias, sendo mais freqüentes, no Brasil, os tipos variantes $\mathrm{S} \mathrm{e} \mathrm{C} \mathrm{e}$ as talassemias alfa e beta, todas na forma heterozigota. Durante 0 ano de 1999, amostras desanguede 506 indivíduos com anemia a esclarecer ou que já passaram por alguma triagem de hemoglobinopatias foram encaminhadas ao Centro de Referência de Hemoglobinas da UNESP, para confirmação diagnóstica e submetidas a procedimentos eletroforéticos, análises bioquímicas e citológicas, para caracterização das hemoglobinas anormais. 0 objetivo do presente estudo foi verificar quais tipos de hemoglobinas anormais apresentam maior dificuldade diagnóstica. As amostras foram provenientes de 24 cidades de doze estados. Os resultados mostraram que 354 indivíduos (69,96\%) apresentaram hemoglobinas anormais, sendo $30 \mathrm{Hb}$ AS (5,93\%), $5 \mathrm{Hb}$ AC $(0,98 \%), 76$ sugestivos de talassemia alfa heterozigota $(15,02 \%)$, 134 sugestivos de talassemia beta heterozigota $(26,48 \%)$ e $109 \mathrm{com}$ outras formas de hemoglobinas anormais $(21,54 \%)$, que incluem variantes raras e interações de diferentes formas de talassemias e hemoglobinas variantes. Concluiu-se que, apesar da melhoria técnica oferecida atualmente e a constante formação de recursos humanos capacitados, as talassemias em sua forma heterozigota ( 210 indivíduos - 41,50\%) são respon sáveis pela maior dificuldade diagnóstica, seguido da caracterização de variantes raras e formas interativas de hemoglobinopatias (109 indivíduos - 21,54\%), sugerindo que se deve aumentar a capacidade de formação de pessoal e as informações a respeito destas alterações genéticas em nossa população.

Rev.bras.hematol.hemoter., 2000, 22(3): 396-403
\end{abstract}

Palavras-chave: Hemoglobinas an ormais, talassemias, diagnóstico

\section{Introdução}

As anemias hereditárias são, das doenças geneticamente determinadas, as mais comuns e compreendem um grupo de condições de considerável complexidade. Alguns tipos de anemias hereditárias são raras e não são importantes do ponto de vista da saúde pública, entretanto, dois grupos se destacam: as doenças hereditárias por alterações das hemoglobinas $(\mathrm{Hb})$ e a deficiência da enzima eritrocitária Glicose-6-Fosfato Desidrogenase (G-6-PD). Aproximadamente 12 a

1 - Biólogo. Mestrando em Genética, UNESP, São José do Rio Preto-SP.

2 - Biomédico. Estagiário do Centro de Referência de Hemoglobinas, Departamen to deBiologia, UNESP, São José do Rio Preto-SP.

3 - Bióloga. Estagiária do Centro de Referên cia de Hemoglobinas, Departamento de Biologia, UNESP, São Josédo Rio Preto-SP.

4 - Professor Titular. Departamen to de Biologia, UNESP, São José do Rio Preto-SP.

5 - Professora Doutora. Departamen to de Biologia, UNESP, São Josédo Rio Preto-SP.

Trabalho realizado no Laboratório de Hemoglobinas, Departamen to de Biologia, UNESP, São José do Rio Preto-SP.

Correspondência para: Cláudia Regina Bonini-Domingos

Laboratório de Hemoglobinas. Departamen to de Biologia, UNESP.

Rua Cristóvão Colombo, 2265. CEP: 15054-000. São José do Rio Preto - SP 
$15 \%$ da população humana é portadora assintomática de uma ou mais formas de anemias hereditárias, notadamente de falcemias, talassemias e deficiência de G-6-PD. Estima-se que estas três alterações causem a mortalidade de três a quatro indivíduos para cada mil recém-nascidos em todo o mundo, induzindo, também, significativa morbidade crônica $(1,2)$.

Milhões de pessoas em todo o mundo carregam, em seu patrimônio genético, hemoglobinas anormais em suas diferentes combinações, com conseqüências que variam de quase imperceptíveis às letais. Sua correta identificação e classificação têm grande importância nas áreas médica, genética e bioquímica. 0 contínuo progresso que se tem obtido em genética humana sobre as alterações que originam doenças hereditárias requer novas estratégias e métodos para detecção de mutantes. No entanto, a extensa heterogeneidade molecular e os vários sítios de mutação em cada gene causavam, até algum tempo atrás, sérias limitações nas pesquisas. Com a introdução da tecnologia de DNA recombinante e PCR (Polymerase Chain Reaction) aumentaram os subsídios para resolução destes problemas. Atualmente, mutações de um determinado gene em particular podem ser detectadas por hibridização e amplificação do alelo específico $(3,4)$.

Os loci das hemoglobinas humanas $(\mathrm{Hb})$ são bem conhecidos e seu estudo fornece dados principalmente em duas áreas: ação gênica e dinâmica dos genes na população. A organização dos genes das globinas humanas tem sido muito estudada, permitindo estabelecer a relação entre a estrutura gênica, o produto final e a sua função (5, 6). O utro fator importante com relação ao gene da globina humana é sua distribuição nas diferentes populações, que resultam em aproximadamente 700 variantes de hemoglobinas e mais de uma centena de tipos de talassemias, fornecendo dados importantes sobre o papel da seleção natural e das migrações humanas $(2,7,8,9,10)$.

O Brasil, país de proporções continentais, caracteriza-se por significativa mistura racial, onde o processo de colonização teve grande influência na dispersão de genes anormais, notadamente de talassemias e falcemias (5). Assim, a distribuição das hemoglobinas anormais, provenientes de formas variantes e talassemias, está intimamente relacionada com as etnias que compõem nossa população. Análises realizadas em indígenas brasileiros não miscigenados revelaram a ausência de hemoglobinas anormais entre diversas tribos de diferentes regiões (11). Recentemente, por meio de técnicas de biologia molecular, analisou-se o gene da globina beta, confirmando-se a ausência de alterações em indígenas da Amazônia (12).

As duas hemoglobinas variantes mais freqüentes na população brasileira, $\mathrm{Hb} \mathrm{S}$ e $\mathrm{Hb}$ $C$, ambas de origem africana, mostram a intensa participação do negro na composição populacional brasileira $(2,3,5)$. Este fato é bem caracterizado nos estudos de prevalência de hemoglobinopatias realizados em diferentes regiões do Brasil $(3,8,13,14)$.

As talassemias do tipo beta diagnosticadas em nossa população têm, por sua vez, significativa origem nos povos provenientes das regiões do mediterrâneo, notadamente de italianos. Da mesma forma como se procederam as distribuições das $\mathrm{Hb} \mathrm{Se} \mathrm{Hb} \mathrm{C}$, as talassemias são mais freqüentes em regiões que tiveram a maior participação da colonização italiana $(3,5,15)$.

A qualidade de pessoal capacitado para o diagnóstico de hemoglobinopatias, aliada à evolução técnica, tem permitido aos laboratórios de rotina realizar exames com maior segurança e contribuir para a melhoria no diagnóstico dessas alterações genéticas.

0 presente estudo teve como objetivo verificar quais os tipos de hemoglobinas anormais apresentam maior dificuldade diagnóstica pelos centros que encaminham amostras de sangue ao Laboratório de Hemoglobinas da UNESP, o qual apresenta atividade de Centro de Referência no diagnóstico destas alterações.

\section{Casuística e Métodos}

Foram utilizadas amostras de sangue, colhidas através de punção venosa e acondicionadas em tubos com anticoagulante (EDTA $5 \%$ ). Muitas das amostras foram prétriadas em seu local de origem, ou apresentavam alguma indicação clínica ou familiar para hemoglobinopatias. 
Os indivíduos foram informados sobre 0 projeto, seguindo-se orientação do Comitê de Ética em Pesquisa e, após autorização, as amostras foram colhidas.

As amostras enviadas ao Centro de Referência de Hemoglobinas foram codificadas e submetidas a triagem de hemoglobinopatias, cujos testes padronizados são:

Resistência Osmótica em solução de Cloreto de Sódio a 0,36\% (16)

Eletroforese em fita de acetato de celulose em $\mathrm{pH}$ alcalino (17)

Análise da morfologia eritrocitária (3)

Amostras apresentando algum tipo de alteração de hemoglobina foram submetidas a testes específicos para confirmação, a saber:

Pesquisa de Corpos de Heinz e Agregados de Hemoglobina $\mathrm{H}$ (18)

Eletroforese em pH ácido (19)

Dosagem de Hemoglobina A2 (17)

Dosagem de Hemoglobina Fetal (20)

Dosagem de Metemoglobina (21)

Focalização Isoelétrica em Gel de Agarose (22)

Eletroforese de Cadeias Polipeptídicas (22)

\section{Resultados}

Durante o ano de 1999 foram analisadas 506 amostras de sangue encaminhadas ao Centro de Referência de Hemoglobinas do Departamento de Biologia da UNESP. O Laboratório de Hemoglobinas, dentro de suas atividades como Centro de Referência de Hemoglobinopatias, auxilia instituições e clínicas particulares na elucidação diagnóstica desta patologia e realiza cursos para a formação de pessoal. Assim, amostras de sangue de indivíduos com anemia a esclarecer ou que já passaram por alguma triagem de hemoglobinopatias são encaminhados para esse Laboratório para confirmação diagnóstica.

No presente estudo, foram analisadas amostras provenientes de vinte e quatro cidades, envolvendo doze estados, conforme mostra a tabela 1.

Do total analisado, 354 amostras de sangue $(69,96 \%)$ apresentaram alguma forma de alteração de hemoglobina. As amostras analisadas foram separadas em dois grupos, aquelas provenientes da cidade de São José do Rio Preto e as enviadas por diversos laboratórios de várias cidades do Estado de São Paulo e outros Estados. A tabela 2 apresenta os resultados obtidos, separados pelos tipos de hemoglobinas encontrados. A figura 1 ilustra a distribuição de diferentes tipos de hemoglobinas, nos dois grupos estudados, com suas respectivas percentagens. Em São José do Rio Preto, observamos que a maior freqüência foi de variantes de hemoglobinas raras ou interações com talassemias (22,37\%), seguidas das talassemias tipo beta heterozigota $(21,05 \%)$ e alfa $(18,42 \%)$. Nas amostras provenientes de outras cidades, encontramos maior freqüência de talassemias do tipo beta $(27,31 \%)$, seguidas de outras hemoglobinas que incluem as variantes raras e formas interativas $(21,30 \%)$.

Tabela 1. Localidades de procedência das amostras analisadas

\begin{tabular}{|c|c|}
\hline CIDADE & ESTADO \\
\hline Salvador & BA \\
\hline Brasília & DF \\
\hline Vitória & ES \\
\hline Rio Verde & GO \\
\hline Três Lagoas & MS \\
\hline Londrina & PR \\
\hline Bom Jesus do Itabapoana & RJ \\
\hline Nova Friburgo & RJ \\
\hline Lagoa Nova & RN \\
\hline Porto Alegre & RS \\
\hline Santa Maria & RS \\
Criciúma & SC \\
\hline Aracaju & SE \\
\hline Bebedouro & SP \\
\hline Campinas & SP \\
\hline Fernandópolis & SP \\
\hline Marília & SP \\
\hline Rio Claro & SP \\
\hline Santa Bárbara do Oeste & SP \\
Santos & SP \\
\hline São Carlos & SP \\
\hline São Paulo Rio Preto & SP \\
\hline
\end{tabular}


Tabela 2. Tipos de hemoglobinas en contrados nos in divíduos analisados pelo Laboratório de Hemoglobinas- UNESP

\begin{tabular}{c|c|c|c|c|c|c|c}
\hline & \multicolumn{7}{c}{ Tipos de Hemoglobinas } \\
\hline Localidade & $\begin{array}{c}\text { AA } \\
\mathbf{n ~ ( \% )}\end{array}$ & $\begin{array}{c}\text { AS } \\
\mathbf{n}(\%)\end{array}$ & $\begin{array}{c}\text { AC } \\
\mathbf{n}(\%)\end{array}$ & $\begin{array}{c}\boldsymbol{\alpha} \text {-Tal. } \\
\mathbf{n}(\%)\end{array}$ & $\begin{array}{c}\boldsymbol{\beta} \text {-Tal. } \\
\mathbf{n}(\%)\end{array}$ & $\begin{array}{c}\text { Outras } \\
\text { Hbs n (\%) }\end{array}$ & $\begin{array}{c}\text { Total } \\
\mathbf{n}(\%)\end{array}$ \\
$\begin{array}{c}\text { São José do } \\
\text { Rio Preto }\end{array}$ & 21 & 08 & - & 12 & 16 & 17 & 74 \\
Outras Cidades & $14,15 \%)$ & $(1,58 \%)$ & $(--)$ & $(2,37 \%)$ & $(3,16 \%)$ & $(3,36 \%)$ & $(14,62 \%)$ \\
& $(25,89 \%)$ & $(4,35 \%)$ & $(0,99 \%)$ & $(12,65 \%)$ & $(23,32 \%)$ & $(18,18 \%)$ & $(85,38 \%)$ \\
TOTAL & 152 & 30 & 05 & 76 & 134 & 109 & 506 \\
& $(30,04 \%)$ & $(5,93 \%)$ & $(0,99 \%)$ & $(15,02 \%)$ & $(26,48 \%)$ & $(21,54 \%)$ & $(100 \%)$ \\
\hline
\end{tabular}

Tabela 3. Número total e percentagem das hemoglobinas anormais de rara freqüência e interações ("outras Hbs") nos en camin hamen tos ao Centro de Referên cia de Hemoglobinas.

\begin{tabular}{|c|c|c|c|}
\hline Tipos deHemoglobinas & \multicolumn{3}{|c|}{ Localidade } \\
\hline (Genótipos) & São José do Rio Preto & Outras Cidades & Total \\
\hline$\alpha / \beta$ Talassemia & $03(2,75 \%)$ & $24(22,02 \%)$ & $27(24,77 \%)$ \\
\hline Hb AS / $\alpha$ Talassemia & $01(0,92 \%)$ & $10(9,17 \%)$ & $11(10,09 \%)$ \\
\hline 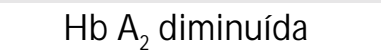 & $02(1,83 \%)$ & $09(8,26 \%)$ & $11(10,09 \%)$ \\
\hline P.H.H.F. & $05(4,59 \%)$ & $06(5,50 \%)$ & $11(10,09 \%)$ \\
\hline Hb SS / P.H.H.F. & $03(2,75 \%)$ & $07(6,42 \%)$ & $10(9,17 \%)$ \\
\hline $\mathrm{Hb} \mathrm{SC}$ & ---- & $07(6,42 \%)$ & $07(6,42 \%)$ \\
\hline $\mathrm{Hb} A \mathrm{D}$ & ---- & $06(5,50 \%)$ & $06(5,50 \%)$ \\
\hline P.H.H.F. / $\alpha$ Talassemia & ---- & $06(5,50 \%)$ & $06(5,50 \%)$ \\
\hline Metemoglobina & ---- & $03(2,75 \%)$ & $03(2,75 \%)$ \\
\hline $\begin{array}{l}\text { Metemoglobina / } \\
\alpha \text { Talassemia }\end{array}$ & ---- & $02(1,83 \%)$ & $02(1,83 \%)$ \\
\hline $\mathrm{Hb} \mathrm{AB}{ }_{2}$ & ---- & $02(1,83 \%)$ & $02(1,83 \%)$ \\
\hline Hb Lepore & $01(0,92 \%)$ & $01(0,92 \%)$ & $02(1,83 \%)$ \\
\hline $\begin{array}{c}\text { Metemoglobina / } \\
\beta \text { Talassemia }\end{array}$ & ---- & $01(0,92 \%)$ & $01(0,92 \%)$ \\
\hline $\begin{array}{c}\text { Metemoglobina / } \alpha \text { / } \\
\beta \text { Talassemia }\end{array}$ & ---- & $01(0,92 \%)$ & $01(0,92 \%)$ \\
\hline Sulfo / Metemoglobina & $01(0,92 \%)$ & ---- & $01(0,92 \%)$ \\
\hline $\mathrm{Hb} \mathrm{SD}$ & ---- & $01(0,92 \%)$ & $01(0,92 \%)$ \\
\hline Hb AS / P.H.H.F. & ---- & $01(0,92 \%)$ & $01(0,92 \%)$ \\
\hline $\mathrm{Hb} \mathrm{CC}$ & ---- & $01(0,92 \%)$ & $01(0,92 \%)$ \\
\hline $\mathrm{Hb} \mathrm{AJ}$ & $01(0,92 \%)$ & ---- & $01(0,92 \%)$ \\
\hline Hb Porto Alegre & ---- & $01(0,92 \%)$ & $01(0,92 \%)$ \\
\hline Hb G Philadelphia & ---- & $01(0,92 \%)$ & $01(0,92 \%)$ \\
\hline $\mathrm{Hb}$ Constant Spring & ---- & $01(0,92 \%)$ & $01(0,92 \%)$ \\
\hline Hb Desconhecida* & ---- & $01(0,92 \%)$ & $01(0,92 \%)$ \\
\hline TOTAL & $17(15,60 \%)$ & $92(84,40 \%)$ & $109(100,00 \%)$ \\
\hline
\end{tabular}




\section{Percentagem dos tipos de hemoglobinas encontradas pelo Centro de Referência nas amostras provenientes de São José do Rio Preto - SP}

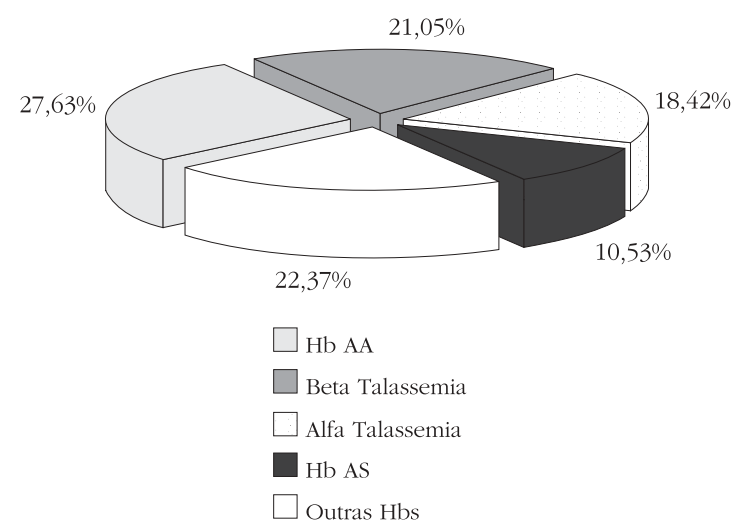

\section{Percentagem dos tipos de hemoglobinas} encontradas pelo Centro de Referência nas amostras provenientes de outras cidades
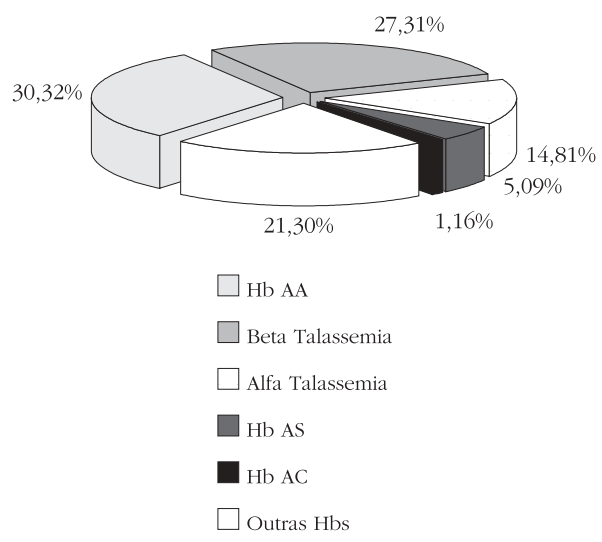

Figura 1. Distribuição percentual dos tipos de hemoglobinas analisados em amostras provenien tes de São José do Rio Preto e ou tras cidades

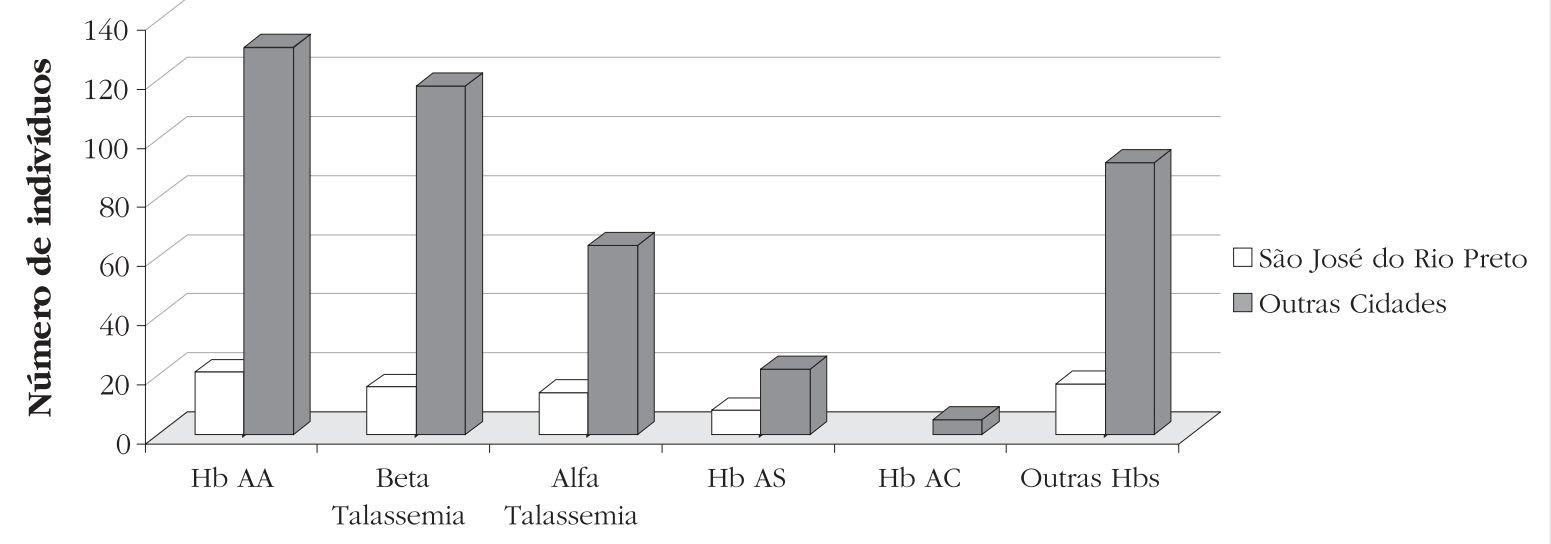

Tipos

Figura 2. Distribuição dos principais tipos de hemoglobinas encontradas em amostras encaminhadas ao Cen tro de Referência de Hemoglobinas

Entre as hemoglobinas anormais foram encontrados diferentes genótipos, evidenciando a grande diversidade genética destas alterações. Nas formas heterozigotas observou-se 5 indivíduos com Hb AC (0,99\%), 30 com Hb AS (5,93\%), 76 sugestivos de portarem talassemia do tipo alfa $(15,02 \%)$ e 134 com talassemia do tipo beta $(26,48 \%)$, entre os dois grupos estudados.

A tabela 3 apresenta os diferentes genótipos para hemoglobinas (classificados como "O utras Hemoglobinas" na tabela 2), separando-se as amostras provenientes de São José do Rio Preto das demais cidades, além da percentagem em relação ao total de amostras analisadas. Dentre as amostras alteradas, encontraram-se diferentes genótipos de hemoglobinas, incluindo variantes raras, quadros de homozigose e interações entre variantes e entre talassemias. 


\section{Discussão}

As hemoglobinas anormais apresentam distribuição variada nas diferentes populações analisadas no mundo todo, que resultam em aproximadamente 700 variantes de hemoglobinas descritas e mais de uma centena de tipos de talassemias, fornecendo dados importantes sobre o papel da seleção natural e das migrações humanas $(7,8,9,14,23)$.

0 Brasil caracteriza-se por significativa mistura racial, com grande influência na dispersão de genes anormais, notadamente de talassemias e falcemias $(3,5)$. A distribuição das hemoglobinas anormais está relacionada com os grupos raciais que participaram na formação da população de cada região (2). Análises realizadas em indígenas brasileiros não miscigenados revelaram a ausência de hemoglobinas anormais entre diversas tribos de diferentes regiões (11), sendo encontradas, assim, nítidas variações regionais na prevalência de hemoglobinas $(2,3,14,24)$.

A alta prevalência de talassemias encontrada no presente trabalho, nos dois grupos estudados, justifica-se por terem sido analisados indivíduos com anemia a esclarecer ou com indicações ou história familiar de hemoglobinopatias.

Entre as formas heterozigotas de hemoglobinopatias, observou-se que a maior dificuldade diagnóstica reside sobre as formas talassêmicas, que juntas - alfa e beta - somam $41,50 \%$ das amostras analisadas. Mesmo com metodologia de fácil aplicação e kits com grande sensibilidade para o diagnóstico dessas alterações, disponíveis no mercado, as dúvidas para finalizar o resultado permanecem. Este fato pode ser explicado por falhas na interpretação dos resultados. Para as talassemias é importante a utilização de um conjunto de técnicas seletivas e de confirmação, aplicadas a cada caso, aliados a histórico clínico e dados hematológicos, na conclusão dos achados laboratoriais.

Apesar de extensivamente pesquisada ao longo dos anos, e facilmente separada por processos eletroforéticos, as variantes de hemoglobina S e C ainda apresentaram dificuldade de caracterização, principalmente no grupo de amostras provenientes de outras cidades. Testes de confirmação para estes tipos de hemoglobinas, como a eletroforese em $\mathrm{pH}$ ácido, podem ser aplicados na rotina laboratorial e servir de suporte par uma conclusão mais segura.

As variantes raras e as formas interativas de hemoglobinas requerem prática do analista e metodologias específicas para confirmação dos resultados. Talvez este seja o motivo de terem sido encontradas tantas amostras com genótipos variados, sem uma conclusão diagnóstica por parte dos analistas que realizam triagens de rotina para hemoglobinas.

A presença de $\mathrm{Hb} \mathrm{A}_{2}$ diminuída pode sugerir ferropenia, onde este estado deve ser afastado para posterior confirmação dos quadros de talassemia. A presença de metemoglobina foi verificada por processos eletroforéticos e sua confirmação como intoxicação e deficiência enzimática confirmada após nova coleta de amostras de sangue, afastando assim, quadros de aumento desta hemoglobina por envelhecimento da amostra. As variantes raras como $A B_{2}$, Porto Alegre, $G$, Constant Spring e forma não caracterizada, foram encaminhadas para estudo estrutural e caracterização molecular.

A figura 2 ilustra a evidente dificuldade no diagnóstico de Hb AS (30 indivíduos) entre as variantes e da talassemia do tipo beta (134 indivíduos) entre as talassemias, além das variantes raras e formas interativas, no grupo de amostras provenientes de outras cidades.

\section{Conclusões}

As talassemias do tipo beta heterozigotas constituem a principal fonte de dificuldade diagnóstica em laboratórios de rotina.

As diferentes metodologias laboratoriais associadas a estudo clínico favorecem 0 diagnóstico das várias formas de talassemias, sendo as técnicas mais utilizadas: eritrograma com índices hematimétricos, resistência globular osmótica em $\mathrm{NaCl}$ a $0,36 \%$, eletroforese em $\mathrm{pH}$ alcalino e ácido, quantificação de $\mathrm{Hb} \mathrm{A}_{2}$ e Fetal e pesquisa citológica de corpos de precipitação.

As variantes de hemoglobina de rara freqüência constituíram o segundo grupo de 
maior dificuldade diagnóstica nas amostras analisadas, evidenciando a carência de formação de pessoal e a dificuldade de implantação de metodologias específicas na rotina.

A avaliação do ferro nas anemias auxilia, sobremaneira, na caracterização de processos talassêmicos.

A combinação genética de variantes de hemoglobina e talassemias fornece dados sobre a intensa diversidade de genótipos para hemoglobinas, cujas implicações, sociais ou clínicas, mostram a importância destas alterações genéticas para a saúde pública, além de evidenciar a nossa miscigenação.

Embora nossa população apresente carência em muitas áreas da saúde pública, cursos de formação de pessoal e metodologias de fácil reprodutibilidade auxiliariam a diminuir o desconhecimento e a falta de diagnóstico adequado destas alterações genéticas.

\section{Abnormal hemoglobins}

Guilherme G. Leoneli, Rodrigo E. Imperial, Daniela P. Marchi-Salvador, Paulo C. Naoum, Cláudia R. Bonini-Domingos

\footnotetext{
Abstract

The human hemoglobins, with genetically defined inheritance patterns, have shown characteristic polymorphic variation within the Brazilian population, depending on the racial groups of each region. They have appeared under the form of hemoglobin variants or thalassemias, the variant types Sand $C$ and thealpha and beta thalassemias being more common, all of them in heterozygote form. During theyear of 1999 , blood samplesfrom 506 individuals, with suspected anemia or that had already passed through hemoglobinopathies screening, were sent to the Hemoglobin Reference Center - UNESP for diagnostic confirmation and submitted to electrophoresis proceedings, biochemical and cytological analyses in order to characterize the type of abnormal hemoglobins. The goal of the present study was to verify which abnormal hemoglobin types show greater diagnostic difficulty. The samples came from 24 cities in twelve states. The results showed that 354 (69.96\%) individuals presented abnormal hemoglobins, 30 (5.93\%) being Hb AS, 5 (0.98\%) being $\mathrm{Hb} \mathrm{AC}, 76$ (15.02\%) suggestive of
}

heterozygote alpha thalassemia, 134 (26.48\%) suggestive of heterozygote beta thalassemia and 109 $(21.54 \%)$ with other forms of abnormal hemoglobin, including rarevariantsand different forms of thalassemias and variant hemoglobin interactions. It has been concluded that, despite the improved techniques currently available and a constant influx of capacitated personnel, the heterozygote form of thalassemias (210 in dividuals - $41.50 \%$ ) is challenging to diagnose, followed in difficulty by rare variant characterization and interactive forms of hemoglobinopathies (109 individuals-21,54\%), suggesting that thecapacity for production of qualified professionals and information about these genetic changes in our population should be increased.

Rev.bras.hematol.hemoter.,2000, 22(3): 396-403

Key words: Hemoglobinopathies, thalassemia, diagnosis

\section{Referências Bibiográficas}

1. W.H.O. - World Health Organization Working Group. Hereditary anaemias: genetc basis, clinical features, diagnosis and treatment. Bulletin of W.H.O., 1982; 60 (5) 643-661.

2. Alvares-Filho, F.O. etal. Variabilidadepolimórfica das hemoglobinas humanas anormais em indivíduos dascidades de Barretose Colina, SP, Brasil. Rev.Bras.Patol.Clin., 1988; 24 (2): 32.

3. Bonini-Domingos, C.R. Preven ção das hemoglobinopatias no Brasil: diversidade genética e metodologia laboratorial. Tese (Doutorado em Ciências Biológicas) - Instituto de Biociências, Letras e Ciências Exatas, Universidade Estadual Paulista - UNESP. 138 p. São José do Rio Preto, 1993.

4. Coutelle, C. New DNA analysis techniques (mini review). Biomed. Biochim. Acta, 1991; 50 (1): 3-10.

5. Naoum, P.C. Anemiasimigrantes: origem das anemias hereditárias no Brasil. Ciência Hoje. 1984; 3 (14): 59-64.

6. Perutz, M.F. Structure and mechanism of hemoglobin. British Med. Bull, 1976; 32 (2): 195-208.

7. Honig, G. R., Adams III, J. G. Human hemoglobin genetics. New York: Springier, 1986. 
8. Salzano, F.M. Hemoglobin variantsin Brazil. In: Winter, W. P. Hemoglobins variants in human population Vol. I. Cap. 8, CRC press, Boca Raton, Flórida, p. 117-130, 1986.

9. Weatherall, D.J., Clegg, J.B. The Thalassemia Syndromes. 3ed. Blackwell Scientific Publications, 1981.

10. Weatherall, D.J. Fortnightly review - The thalassemias. B.M.J. 1997; v. 314.

11. Naoum, P.C. et al. Concentration of haemoglobin $A_{2}$ and fetal in brazilian indians relationship between these haemoglobins and malaria. Ciência Hoje 1979; 31: 188-190.

12. Guerreiro, J.F., et al. Beta globin gen e cluster haplotypes in Yanomama Indians from Amazon region of Brazil. Hum. Genet, 1982; 89: 629-631.

13. Hut, M. H., Salzano, F. M., Adams, J. Hb F levels, longevity of homozygotes and clinical course of sickle cell anemia in Brazil. Amer. J. Med. Genet, 1983; 14: 669-676.

14. Naoum, P. C. Diagnóstico deHemoglobinopatias. São Paulo: Sarvier, 1987.

15. Ramalho, A. S. A talassemia minor como causa da anemia no Estado de São Paulo.

Rev. Bras. Pat. Clin., 1986; 22: 32-38.

16. Silvestroni, E., Bianco, I. Screening for microcytemia in Italy: analysis of data collected in the past 30 years. Am. J. Human Genet, 1975; 27: 198.

17. Marengo-Rowe, A.J. Rapid electrophoresisand quantification of hemoglobin on cellulose acetate. J. Clin. Pathol., 1965; 18: 790-792.
18. Papayannopoulos, R., Stamatayannopoulos, G. Stains for inclusion bodies. In: Standardization of laboratory reagents and methodsfor detection of haemoglobinopathies. Atlanta, Hew Publications, 1974.

19. Vella, F. Acid agar electrophoresis of human hemoglobin. Am. J. Clin. Pathol, 1968; 49: 440.

20. Betke, K., Marti, N.R., Schlicht, I. Estimation of small percentages of phoetal hemoglobin. Nature, 1959; 184: 1877.

21. Gerald, P.G. The methemoglobinemias, In: Stamburu, J.B., Wyngaarden, J.B. and Fredickson, D. S. (Ed.). Metabolic basis of inherited disease. New York: Mac Graw Hill, 1966. p. 1069.

22. Naoum, P.C. Eletroforese, Técnicas e Diagnósticos. São Paulo: EditoraSantos, 1990.

23. IHIC - International Hemoglobin Information Center. Alphabetical Hemoglobin Variant List. Hemoglobin. 1996; 20: (3): 313-335.

24. Bonini-Domingos, C.R. Prevalência de hemoglobinas anormais, fenótipos de haptoglobinas e quantificação de hemoglobinas A2 e Fetal em portadores de Doença de Chagas. Dissertação (Mestrado em Ciências Biológicas) - Instituto de Biociências, Letras e Ciências Exatas, Universidade Estadual Paulista - UNESP. 143 p. São José do Rio Preto, 1990.

Recebido: 09/02/2000

Aceito: 18/10/2000 